\title{
Gaan Mawina, le Marouini (haut Maroni) au cœur de l'histoire des Noirs marrons Boni/Aluku et des Amérindiens Wayana
}

Gaan Mawina (Marouini): elements for a mapping of the history of the Boni/ Aluku Maroons and Wayana Amerindians on Upper Maroni

\section{Marie Fleury}

\section{OpenEdition}

Journals

Édition électronique

URL : http://journals.openedition.org/ethnoecologie/3534

DOI : $10.4000 /$ ethnoecologie.3534

ISSN : 2267-2419

Éditeur

Laboratoire Eco-anthropologie et Ethnobiologie

Référence électronique

Marie Fleury, « Gaan Mawina, le Marouini (haut Maroni) au cœur de l'histoire des Noirs marrons Boni/ Aluku et des Amérindiens Wayana », Revue d'ethnoécologie [En ligne], 13 | 2018, mis en ligne le 21 juin 2018, consulté le 19 avril 2019. URL : http://journals.openedition.org/ethnoecologie/3534 ; DOI 10.4000/ethnoecologie.3534

Ce document a été généré automatiquement le 19 avril 2019

Revue d'ethnoécologie est mis à disposition selon les termes de la licence Creative Commons Attribution - Pas d'Utilisation Commerciale - Pas de Modification 4.0 International. 


\section{Gaan Mawina, le Marouini (haut Maroni) au cœur de l'histoire des Noirs marrons Boni/Aluku et des Amérindiens Wayana ${ }^{1}$}

Gaan Mawina (Marouini): elements for a mapping of the history of the Boni/

Aluku Maroons and Wayana Amerindians on Upper Maroni

\section{Marie Fleury}

In Memoriam

En hommage à Jean Hurault, Géographe français qui a fait un travail d'ethnographie remarquable chez les Boni/Aluku et les Wayana dans les années

1960 , et en remerciement pour la confiance accordée en nous confiant ce travail en 1999.

En mémoire des Capitaines Charles Cazal et Louis Topo, qui nous ont quittés respectivement en 1999

et en 2016, en témoignage de notre amitié et profond respect.

\section{Introduction}

1 L'histoire des Aluku nommés également Boni remonte aux révoltes d'esclaves au Surinam au XVIII ${ }^{\mathrm{e}}$ siècle où les Africains déportés d'Afrique pour des travaux serviles s'échappaient des plantations hollandaises pour fuir les conditions intolérables de vie sur les plantations esclavagistes. Dès le début de la colonisation des esclaves s'enfuyaient des plantations. Mais le phénomène prit de l'ampleur quand les maîtres les envoyèrent se cacher en forêt, pour éviter de payer l'impôt de capitation. Beaucoup ne revinrent pas, profitant de l'occasion pour fuir définitivement en forêt. Manquant de tout, ils lançaient des assauts sur les plantations pour voler des vivres, des armes, et libérer de nouveaux 
esclaves. Les Hollandais excédés par ces attaques incontrôlables et de plus en plus nombreuses finirent par se résigner à signer des contrats de paix avec les rebelles : en contrepartie ceux-ci devaient ramener tous les nouveaux fugitifs. C'est ainsi que fut accordée la liberté aux groupes Ndjuka, Saramaka, Paramaka et Matawai. La plupart des groupes ont émergé à la fin du XVII et au début du XVIII ${ }^{\mathrm{e}}$, (Saramaka, Matawai, Ndjuka...). Les Boni furent un des derniers groupes à se former, au milieu du XVIII ${ }^{e}$ siècle, et ce sont les épisodes vécus sur le Marouini, affluent du Maroni où vivaient aussi des Amérindiens Wayana que cet article tente de documenter. La carte est ici un objet de rencontres, à la fois pour placer des données, controversées ou non, obtenues de plusieurs sources orales et écrites à différentes époques et pour travailler à son élaboration en réunissant Wayana et Noirs marrons sur les lieux importants d'une histoire commune.

\section{Contexte de l'étude}

2 Cet article retrace une partie de l'histoire des Boni en croisant différents types de sources : des documents inédits sous forme de notes de travail du géographe Jean Hurault (cf. encart), confiés en même temps qu'un projet de cartographie sur le terrain, un récit de voyage mené sur les lieux de cette histoire avec un groupe d'Anciens connaissant la tradition orale, et la littérature sur la région avec notamment les travaux des historiens et anthropologues spécialistes de la Région. C'est en croisant ces différentes sources, chacune avec des limitations et des intérêts particuliers, que nous avons réalisé, pour l'essentiel collectivement durant la remontée du fleuve, la carte des lieux principaux de cette histoire des Wayana et des Boni, telle que présentée dans cet article.

3 En regrettant de ne pas avoir effectué lui-même un travail de reconnaissance des emplacements des anciens villages aluku du Marouini, Jean Hurault me demanda de réaliser un relevé de ces anciens lieux d'habitat, accompagnée d'Anciens savants aluku et wayana. Pour réaliser cette mission, en juillet 1999 (Fleury 1999a), j'ai affrété une pirogue à Monsieur Kawet Sintaman, qui a souhaité être accompagné de sa femme, elle-même fille du capitaine Haïwé et nièce du Gaan Man wayana. J'avais demandé à Monsieur le capitaine Cazal de nous accompagner, sur les recommandations de Monsieur J. Huraut, car il connaissait bien l'histoire de son peuple et du territoire. Il a accepté à condition d'être accompagné de son petit-fils qui l'aiderait au quotidien, car il était déjà très âgé. Participaient également à la mission Monsieur Topo, obiaman de la communauté Aluku avec qui je travaillais depuis de nombreuses années, et Monsieur Haiwë, capitaine du village de Twenké, et beau-frère de l'actuel Gaan Man Amaïpoti (héritier direct d'un acteur de cette histoire) qui pourrait nous renseigner sur les anciens emplacements des villages wayana.

4 L'expédition se déroula du 21 au 26 juillet 1999, et nous a permis de cartographier les emplacements d'une quarantaine de toponymes aluku et wayana sur le Marouini (Gaan Mawina) sur un fond de carte obtenu d'une carte IGN (Figure 2) en même temps que de recueillir et de comparer les différentes versions de l'histoire contées chez les Aluku et chez les Wayana (Fleury 1999b). 
Figure 1 : Village Apalai sur le bas Marouini pendant la mission de cartographie en 1999

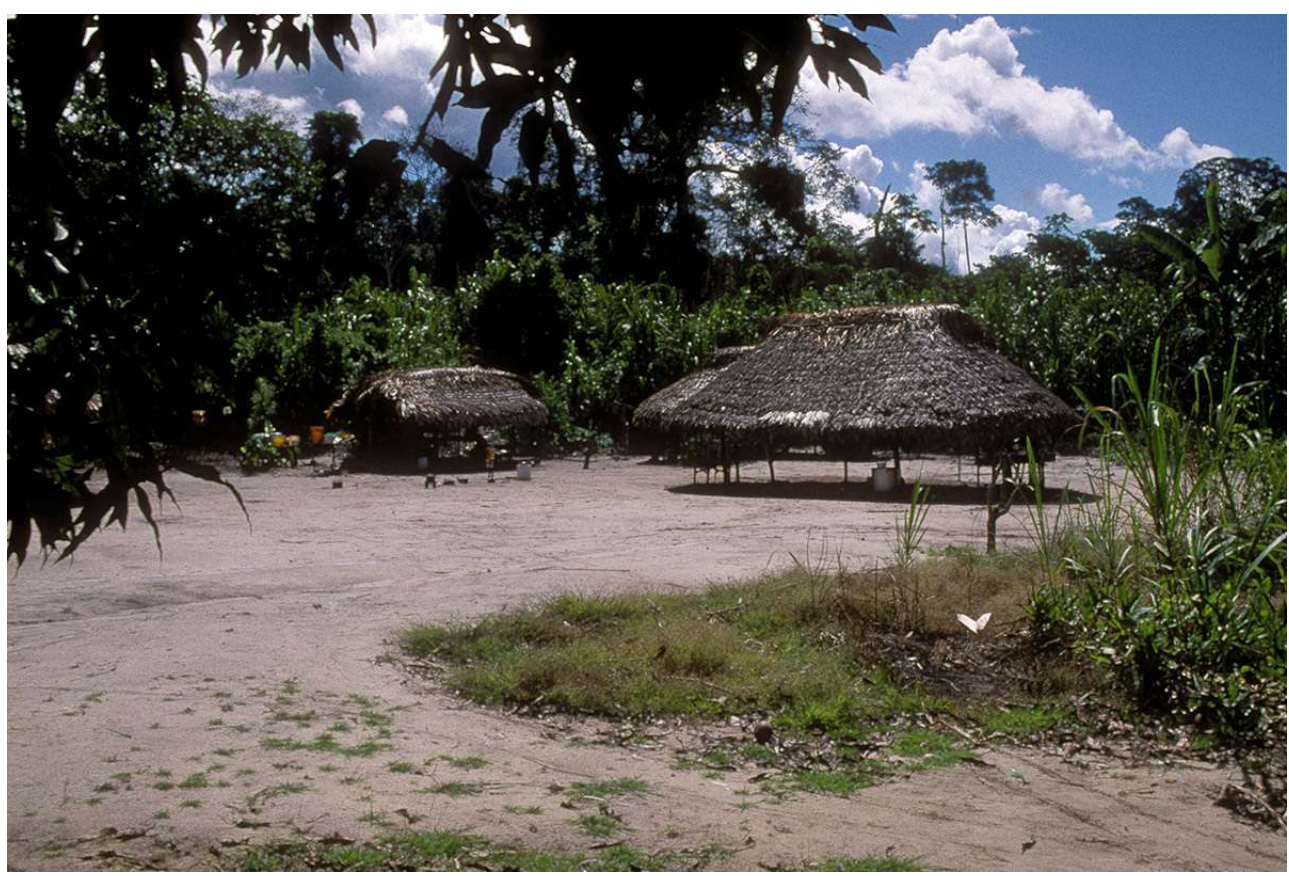

(c) M. Fleury

Figure 2 : Occupation ancienne du Marouini à la fin du XVIII siècle

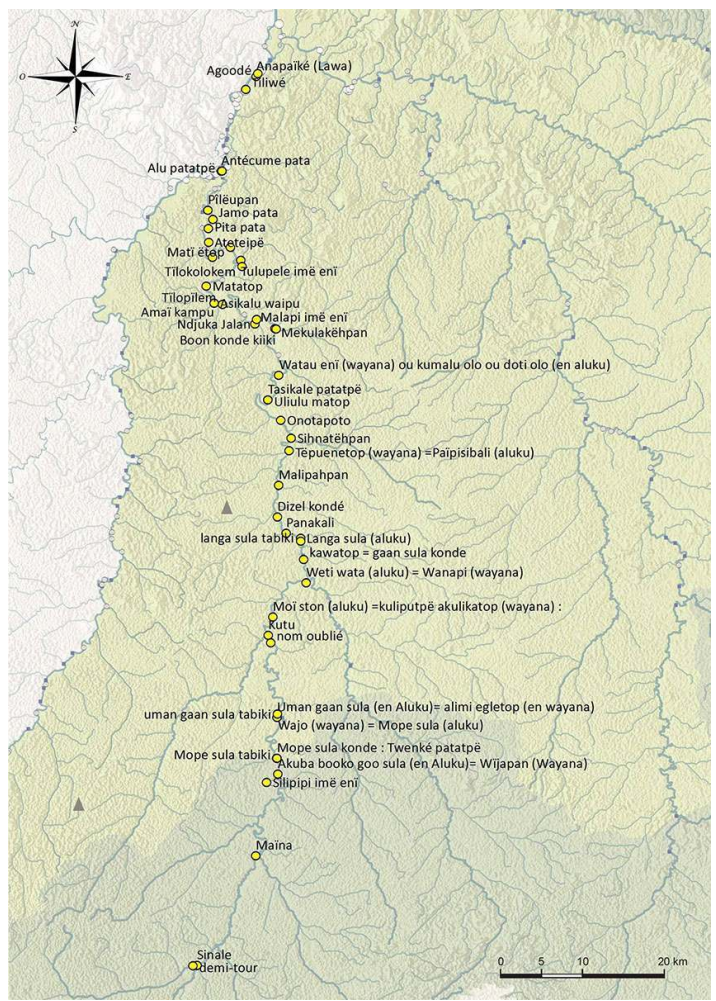

5 À cette époque (fin $\mathrm{du} \mathrm{xx}^{\mathrm{e}}$ siècle), les méthodes de cartographie dites participatives n'étaient pas encore très développées, et notre travail relevait davantage d'une relation chercheur/informateur classique, avec de forts liens d'amitiés et de véritables moments 
de convivialité et de partage. Les membres de l'équipe de travail, choisis comme cela a été précisé ci-dessus, étaient nécessairement peu nombreux et ne pouvaient (ni ne prétendaient) être représentatifs de l'ensemble de la société locale. En outre, il faut souligner que les enjeux territoriaux n'étaient pas les mêmes qu'ils pourraient l'être à d'autres époques, y compris plus contemporaines comme nous verrons plus loin: il s'agissait avant tout d'un travail collectif d'enregistrement de savoirs spécifiques sur les lieux et les événements du passé par un petit groupe de passionnés, travail au cours duquel le chercheur occupe d'abord une place de scribe (en transcrivant sur la carte et le papier des informations transmises oralement).

6 La scolarisation a énormément modifié les relations entre chercheurs et informateurs, ainsi que les postures des chercheurs et les contextes dans lesquelles les recherches sont réalisées. Pour ce qui nous concerne, ceux qui étaient des traducteurs sont devenus des collaborateurs à part entière, puis pour certains, des co-auteurs comme cela a été le cas pour notre travail de cartographie participative avec les Wayana sur le Litani (Fleury et al. 2016).

7 Si les méthodes participatives prennent le pas en ethnographie dans les travaux avec les populations autochtones, c'est aussi parce que celles-ci ont elles-mêmes acquis certaines techniques et des enseignements académiques tout en revendiquant une place dans les débats scientifiques et politiques actuels. Entre le travail de cartographie réalisé en 1999 et celui de 2013, la différence essentielle, vient du fait que le premier était une demande de Jean Hurault (géographe spécialiste du Maroni), alors que dans le deuxième cas, la demande venait des Jeunes de la communauté (habitants des villages du Litani) intéressés à mieux connaître leur histoire et leur territoire. Ils se sont donc beaucoup investis dans les recherches sur le terrain, ce qui a permis de travailler avec de nombreuses personnes et d'accéder à beaucoup de témoignages d'Anciens. La relation intergénérationnelle dans ce cas est extrêmement importante et intéressante, les Anciens étant eux aussi ravis de voir les Jeunes s'intéresser à leur histoire. 
Figure 3 : Passage d'un saut sur le Marouini en 1999

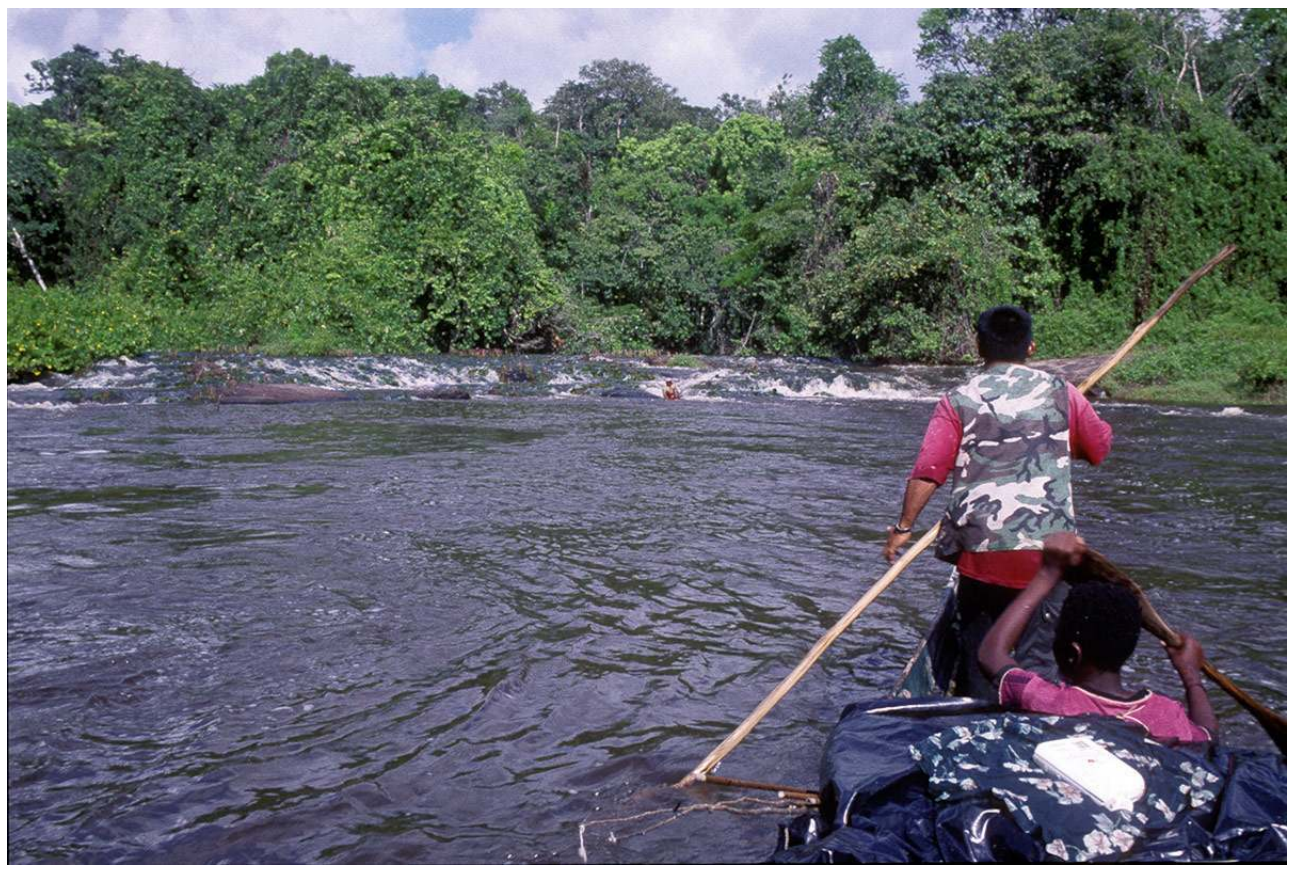

(c) M. Fleury

8 La méthode de cartographie avec un retour sur les lieux du passé présente également un grand intérêt par rapport à des enquêtes plus classiques en ethnologie d'enregistrements de témoignages au village. En effet, les lieux ravivent la mémoire des Anciens, d'autant plus que la confrontation entre différents descendants des acteurs de l'histoire pouvait être faite in situ au vu de la composition de l'équipe. Les soirées au campement étaient également l'occasion idéale d'échanges autour du feu, et les conditions étaient donc propices à la discussion et aux souvenirs. 
Figure 4 : Repas au village Apalai sur le bas Marouini (Topo et Haiwé), durant la mission en 1999

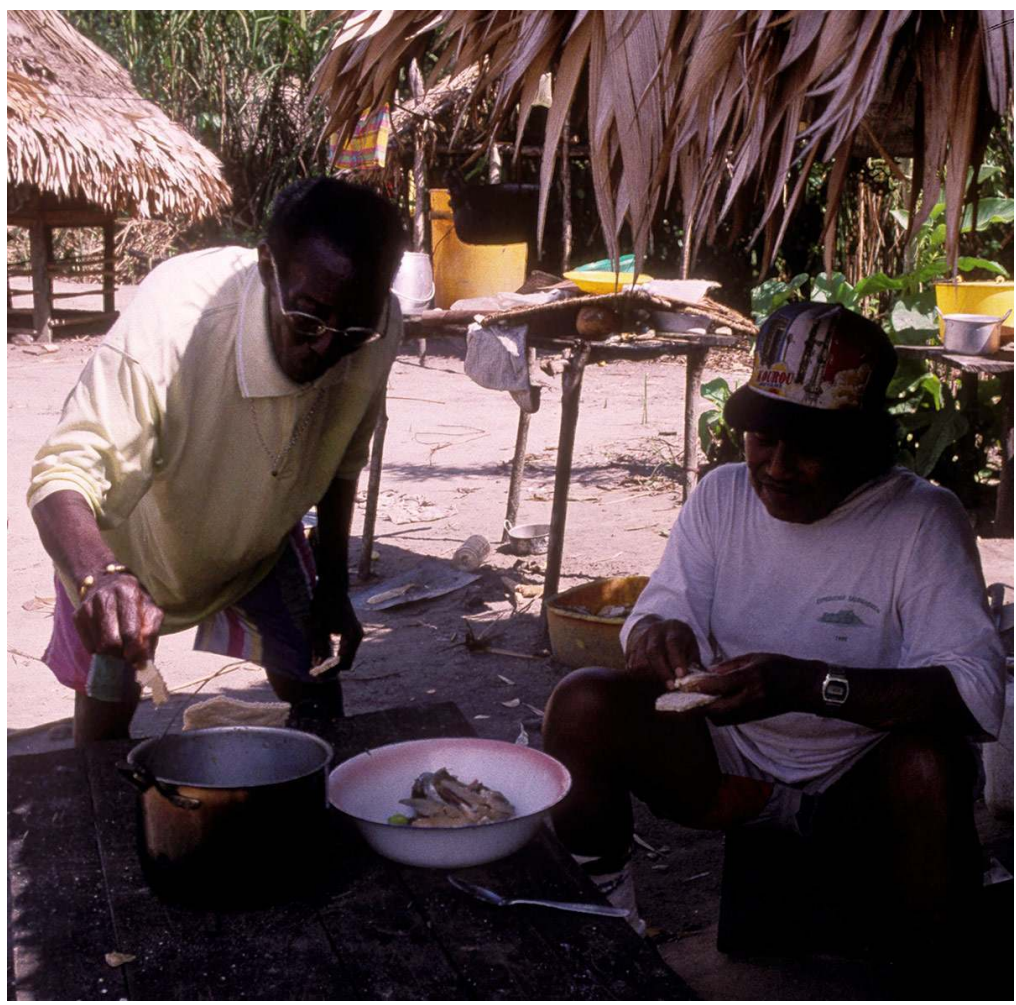

(c) M. Fleury

Bien-sûr cette cartographie "vécue » en récit de voyage s'est enrichie également à travers une confrontation avec les sources écrites : certains éléments ayant pu disparaître de la mémoire orale, ou bien tout simplement être omis, volontairement ou pas. La datation est également plus facile avec les témoignages datés des explorateurs et des chercheurs. C'est donc une synthèse des documents écrits et de la mémoire orale, qui nous permet de fournir des cartes historiques du peuplement de la région (Figures 2, 5, 6 et 7). 
Figure 5 : Migrations historiques des Boni sur le Maroni au XVIII siècle, sur un fonds de carte contemporain

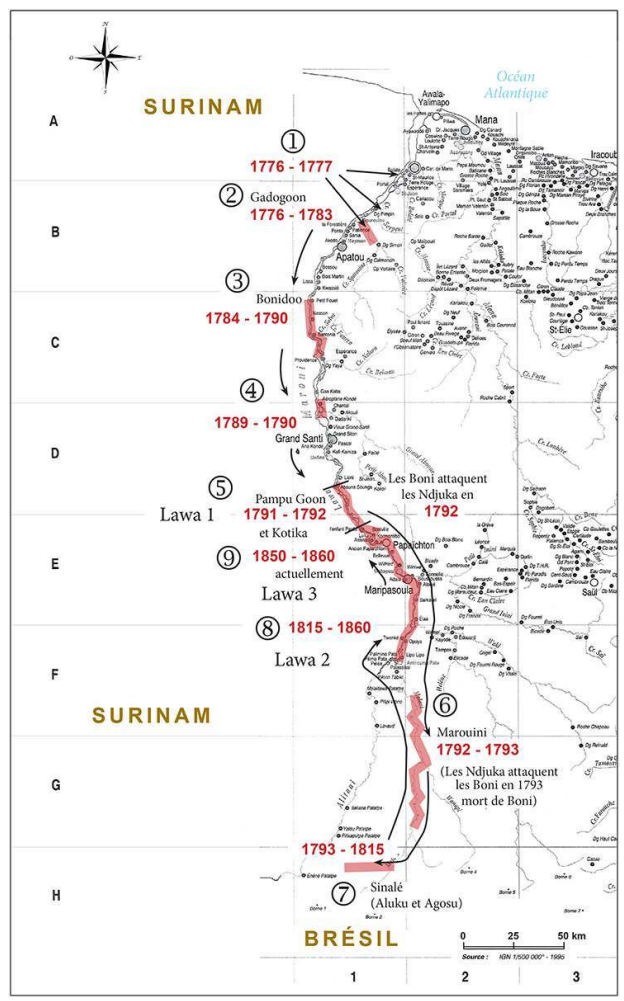

Figure 6 : Occupation des rivières Alitani et Marouini à la fin du XVIII siècle-début XIX ${ }^{e}$ siècle

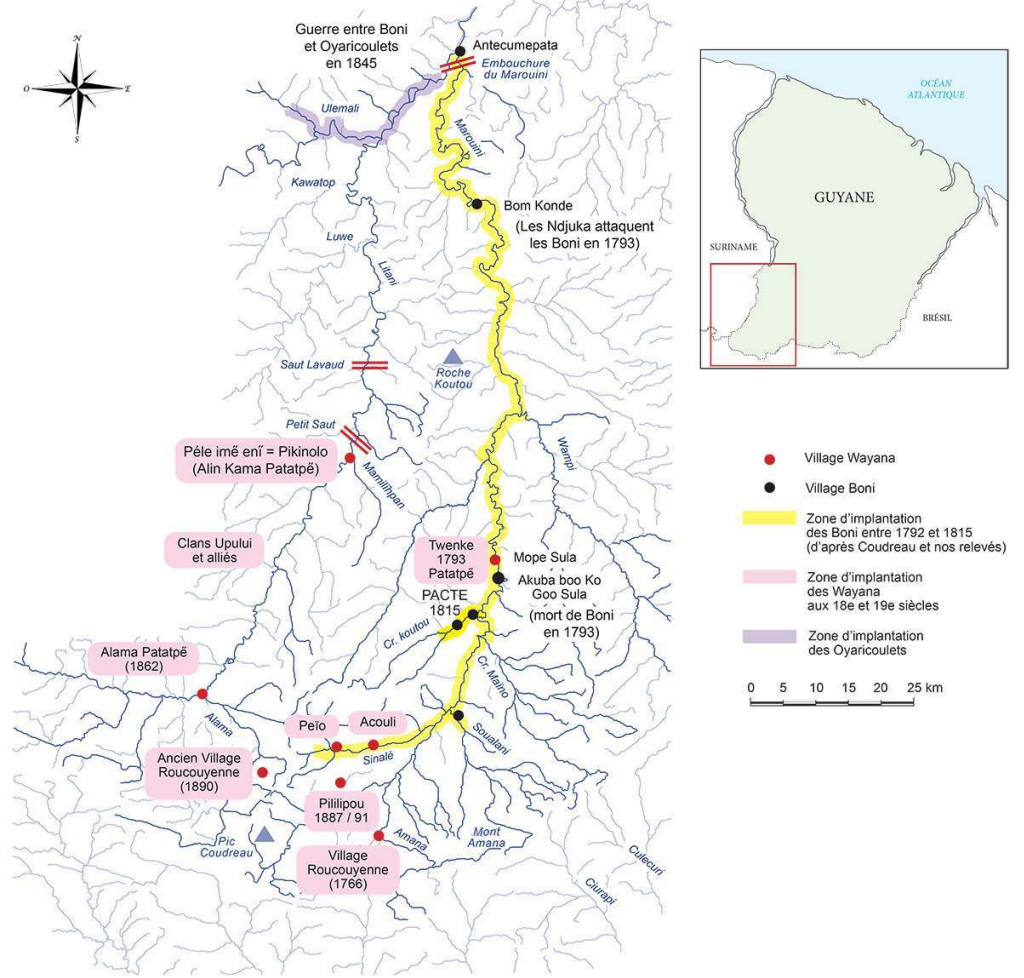


Figure 7 : Chronologie de l'installation des villages Boni le long du Maroni à la fin du XVIII siècledébut XIX siècle, selon le récit de Cazal (1950) sur un fonds de carte contemporain

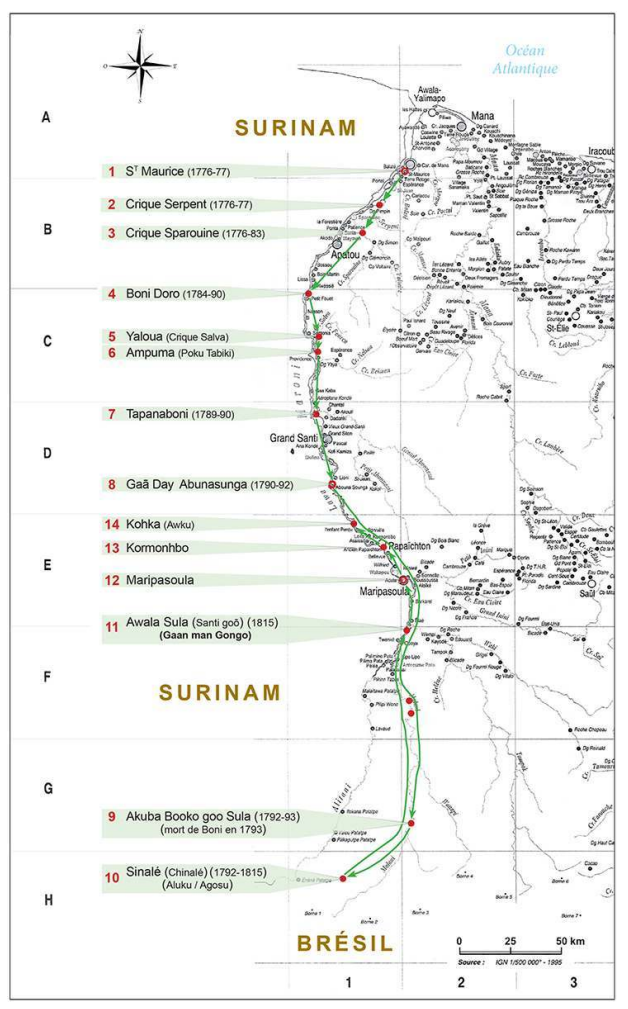

\section{L'histoire mouvementée des Noirs marrons Boni (Aluku)}

Les Boni sont un des six groupes de Noirs marrons ${ }^{2}$ vivant en Guyane française et au Surinam, les autres étant les Ndjuka, les Paramaka, les Saramaka, les Matawai et les Kwinti. La population Boni, dont la démographie a explosé au cours des quarante dernières années, est estimée à environ 11000 personnes $^{3}$ en 2014 (Price 2013b), vivant pour la majorité d'entre eux sur le long du Lawa (haut Maroni) et du bas Maroni (Apatou et St Laurent du Maroni) en Guyane française.

\section{Origine africaine}

Les Noirs marrons sont des descendants d'esclaves rebelles qui ont fui les plantations hollandaises aux XVII ${ }^{e}$ et XVIII ${ }^{e}$ siècles. On peut localiser leur origine africaine sur la côte occidentale de l'Afrique : la plupart des esclaves transportés au Surinam par la traite, provenaient de la Côte des esclaves, englobant la Côte d'Or (actuel Ghana), le Dahomey (actuel Bénin) et le Nigeria (Van der Kuyp 1961). Étant donné les nombreux brassages de populations qui ont eu lieu, il est cependant difficile de préciser les lieux d'origines africaines des Noirs marrons. Certains traits culturels peuvent toutefois être retrouvés : ainsi à Haïti, et dans le Nord brésilien, domine la culture Fon du Dahomey, et dans les petites Antilles françaises, celle des Kromanti de la Côte d'or (actuel Ghana). Chez les Noirs marrons des Guyanes française et hollandaise on retrouve des traits de la culture 
Fanti-ashanti du Ghana, en particulier dans les domaines de l'organisation sociale et des croyances religieuses (Bastide 1967), et une influence bantoue en ce qui concerne la linguistique (Price 2013a).

\section{Marronage (Lowe ten)} libérés après leur période de service. Ceux-ci jouèrent un rôle important dans la guerre contre les Marrons, notamment pour la prise de Buku, place forte des Boni, située au milieu de marécages. C'est suite à cette défaite, que les Boni traversèrent le Maroni, entre août 1776 et janvier 1777, et s'installèrent du côté français, sur le bas Maroni. Par sécurité, ils opérèrent en différentes phases:

- Un groupe partit de la crique Wana et de la pointe Galibi et traversa vers la crique Coswine.

- Un groupe passa par Bigiston (en amont de l'actuel Albina) et arrivèrent à Lawalawa, entre la crique Balaté et l'île Portal, près de l'actuelle ville de St Laurent du Maroni.

- Un groupe tenta de s'installer sur la crique Boni (Boni kiiki), mais n'y resta pas,

- et enfin un groupe s'installa près de la crique Yooka kiiki en amont de l'île Lemikibon ( Lemiki bon tabiki) (Moomou 2013).

19 Au lieu-dit Pempin, sur la rive française, ils organisèrent une grande réunion (gaan obia), où ils prirent la décision de remonter le Maroni, notamment sur la crique Sparouine, un affluent de la rive droite, et en amont de celle-ci. 
la crique Sparouine et le long du Maroni, jusqu'à l'île Langa tabiki (Figure 5) :

- Boni fonde Gadogoon (1777-1783) sur la crique Sparouine, non loin du village de Mapika (autre village de Noirs marrons fondé dans les années 1760). Il y reste jusqu'en 1783. Il est peuplé d'une centaine de personnes avec des femmes et des jeunes.

- Koomanti Kodjo fonde le village de Langa tabiki ( 40 femmes et 25 hommes) : juste en amont de Boni-doo.

- Puja du clan Lapaix quitte son village de la crique Wana, détruit par le colonel Fourgeoud, traverse en janvier 1777 et fonde un village à Assalobi Tabiki.

Les Boni établissent alors des contacts avec les Indiens Galibi voisins (Kali'na) qui sont des partenaires commerciaux mais aussi des intermédiaires avec l'administration coloniale française (Moomou 2013).

Les Français de l'époque les estiment alors de 8 à 10000 individus ${ }^{7}$, alors qu'en réalité ils ne sont que quelques centaines. La colonie française étant alors relativement pauvre, les nouveaux arrivants sont acceptés, en espérant y trouver une source de main d'œuvre, tout en craignant des conflits avec les forces hollandaises voisines (De Groot 1984), mais aussi que leur liberté soit un «mauvais » exemple pour leurs propres esclaves. Cette ambiguïté restera longtemps caractéristique de l'attitude des Français vis-à-vis des Boni (Hurault 1960).

Dès le mois d'octobre 1777, les Ndjuka, inquiets de voir ces nouveaux arrivants sur leur territoire, lancent une attaque contre les Boni. La paix revient à la fin de l'année. Il s'en suit une période de réconciliation entre Boni et Ndjuka, avec des échanges matrimoniaux. Boni obtient ainsi la fille d'un chef Ndjuka ${ }^{8}$ comme épouse.

torités françaises tentent plusieurs approches pour les faire installer sur la Mana, mais les Boni ne leur accordent pas confiance et n'y répondent pas favorablement (Moomou 2013). ou goon/abattis) à Gaan day (au saut Abouna Sounga), attaquent les Ndjuka à Animbaw. S'en suit une guerre sans merci et les Boni se réfugient sur le Marouini.

\section{L'épopée du Marouini}

Entre 1792 et 1815, les Boni se réfugient sur la rivière Marouini, affluent du Maroni, où ils rencontrent les Wayana, installés aux environs de sa source depuis le XVIII ${ }^{\mathrm{e}}$ siècle (Fleury et al. 2016). C'est cette période de leur histoire, très mal connue, que nous abordons à travers la cartographie des anciens lieux de vie wayana et aluku de l'époque. (Figures 2 et 6). 

dans ses retranchements sur le Marouini en février 1793. Boni se fait assassiner par Bumbi, chef Ndjuka, qui réussit à le tuer pendant son sommeil, et le décapite pour toucher la prime des Hollandais. Toutefois la pirogue coule durant le voyage de retour (au saut Dagu ede ${ }^{11}$, selon la version de Cazal, au saut Simayé, selon d'autres versions) et il est obligé de la remplacer par une autre tête (cf. encadré). La plupart des Boni sont tués dans cette attaque des Ndjuka sur le Marouini, les villages sont brûlés (d'où le nom Boon konde (boon/brûlé; konde/village). Et les Wayana, qui se souviennent de cet épisode sanglant, nous ont montré le lieu-dit Ndjuka Jalan (Le boucan des Ndjuka) où ceux-ci auraient torturé et boucané (brûlé ?) les Boni. (Figures 2, 5, 6 et 7).

Figure 8 : Embouchure de la crique Sinale (Chinalé) affluent du Marouini durant la mission en 1999

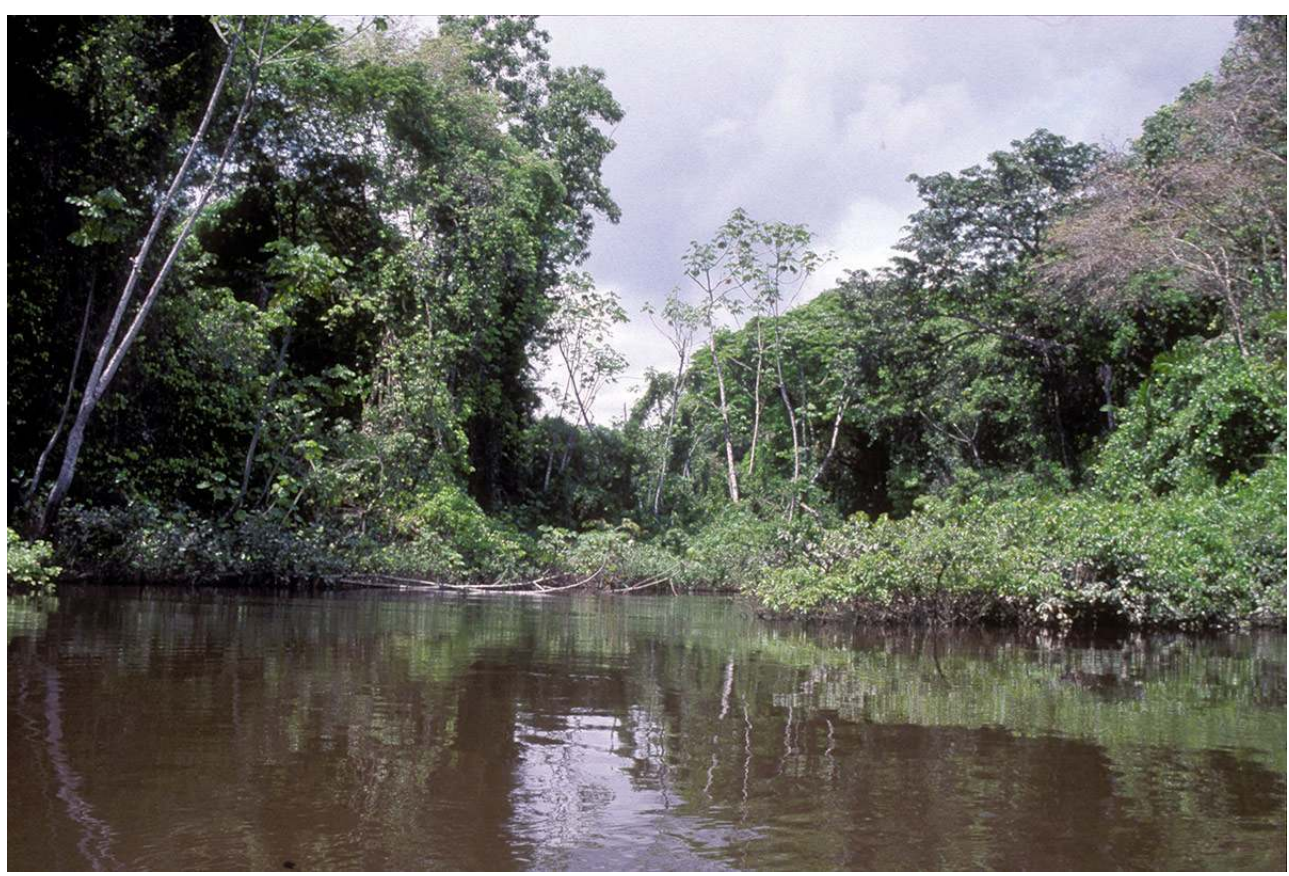

(c) M. Fleury

Selon les versions, certains survivent ou non à ce terrible épisode. Les Boni racontent que seuls Aluku et le fils de Boni, Agosu, réussirent à s'enfuir avec quelques rescapés, plus en amont sur le Marouini, sur la crique Sinalé, où vivent déjà des Wayana. Ceux-ci nous ont raconté leurs versions de l'histoire dans laquelle tous les Boni ont été exterminés, sauf une femme enceinte (et un enfant selon les versions). Les Wayana la cachèrent dans une jarre à cachiri, pour la sauver des Ndjuka. C'est cette femme ${ }^{12}$ qui aurait reconstitué le peuple Boni, par un inceste fondateur. Cette version de l'histoire des Boni par les Wayana est intéressante car elle rappelle un des mythes de la création. En effet, le dieu Mopo, deuxième personnage du panthéon wayana, est lui-même né dans une jarre à cachiri (oha ) dans la mythologie wayana (Chapuis \& Rivière 2003). La jarre à cachiri symbolise ici le ventre maternel, de même que la fermentation de la bière peut être assimilée à une gestation (Fleury 2010). À travers ce mythe les Wayana réinventent l'origine du peuple Boni, le fait entrer dans leur cosmogonie et lui donnent une place dans leur système d'alliance.

Revue d'ethnoécologie, 13 | 2018 
Dans la tradition orale des Boni, c'est Aluku qui aurait permis d'éviter l'anéantissement du groupe, grâce à sa fuite en amont du Marouini. C'est pourquoi les Aluku ne se désignent plus eux-mêmes Boni, mais Aluku nenge, car ils sont tous des descendants d'Aluku (Aluku pikin). Toujours d'après les récits des Boni/Aluku sur leur propre histoire, ils n'auraient jamais vécu chez les Wayana, mais dans des villages voisins (Topo comm. pers.).

Une paix fut scellée entre Wayana et Aluku sur la crique Kutu (un affluent du Marouini) en 1815. Selon Coudreau ce pacte de paix et d'amitié fut contracté entre le père de Twenké (Ouané) et le nouveau Gaan Man Boni : Gongo (Coudreau 1893 : 104). Il s'agit certainement d'un sweli (mélange des sangs suivi de l'absorption du breuvage par les deux Parties) tel qu'il est souvent décrit par divers auteurs. Cet acte symbolique ne signifie pas que les Wayana et les Aluku étaient précédemment en guerre, mais vient renforcer leurs liens d'amitié avant que les Aluku ne quittent la rivière. Depuis cette union, ils se considèrent comme "Alukuyana", (Aluku et Wayana soudés) soulignant leurs liens d'amitiés et l'unité de leurs peuples. Certains auteurs y voient surtout une sorte de captation des Wayana par les Aluku, pour les inclure dans leur monde comme un prolongement d'eux-mêmes, les Wayana devenant «leurs Indiens » avec qui ils vont établir des relations commerciales privilégiées (Dupuy 2015 : 274).

Cette période de contact étroit entre les Wayana (qui ont certainement dû accueillir dans un premier temps les rescapés, avant qu'ils ne reconstruisent leurs propres villages) et les Aluku a été l'occasion de multiples échanges culturels. Ainsi les Wayana maitrisaient la culture et la transformation du manioc amer et les Boni ont certainement bénéficié de cette connaissance (ils cultivaient surtout du riz et autres plantes alimentaires durant le marronnage, car le riz est beaucoup moins long (trois mois) à récolter que le manioc amer (un an) (Fleury 1996). D'autres influences culturelles sont également à souligner, notamment dans certains rituels (Fleury 1991).

Parallèlement les Boni ont appris aux Wayana, la technique de construction des canots monoxyles, qui vont leur permettre de passer les grands sauts et d'occuper le Litani. En effet avant leur rencontre avec les Boni, ils ne construisaient que des canots précaires en écorces pour traverser les rivières (Coudreau 1893). Cette période de cohabitation a donc eu une forte influence sur les deux sociétés qu'étaient les Wayana et les Aluku de l'époque. 
Figure 9 : Passage d'un saut du Litani par Epoya, Passikili et un troisième homme en 1964

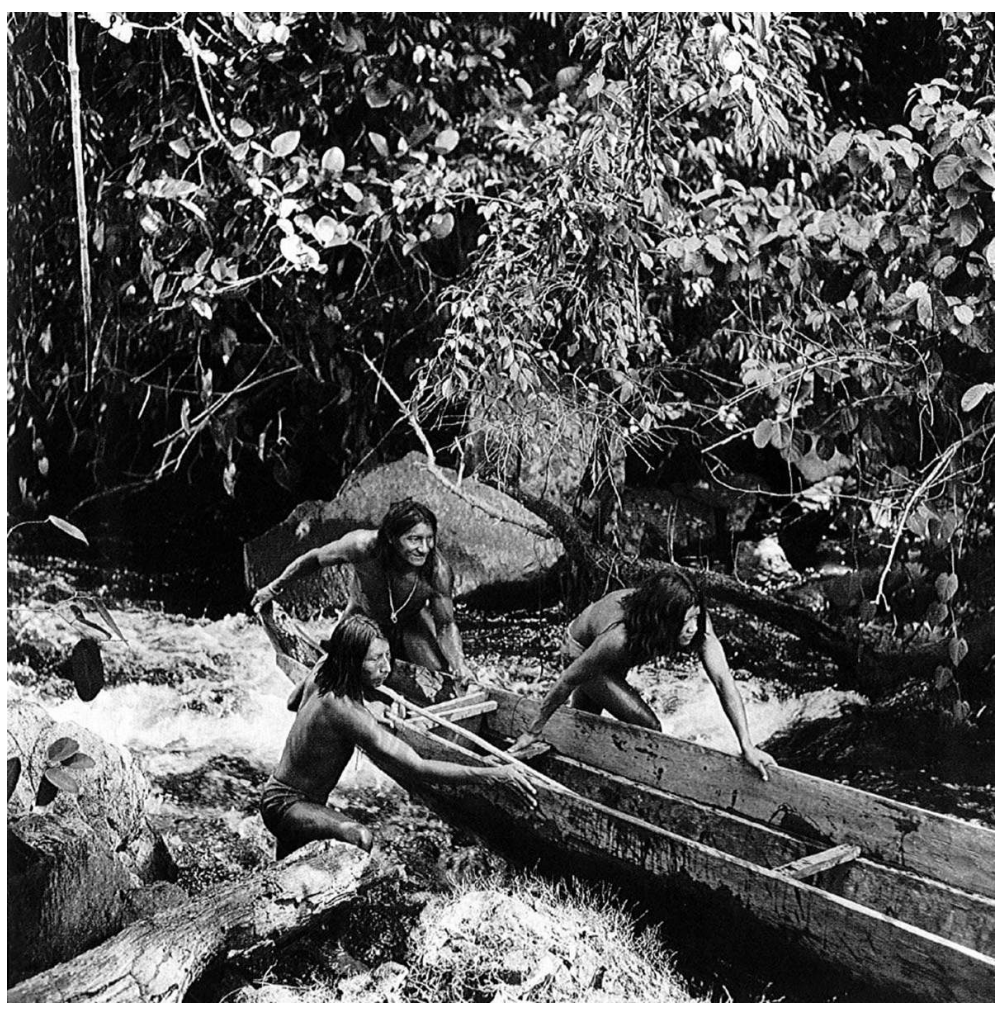

(c) J. Hurault (Hurault et al. 1998)

\section{La présence des Wayana sur le Marouini dans les textes écrits}

mont Amana et se jetant dans le Marouini) :

«cette crique est presque sans eau et peu profonde, sur ses bords pousse le cacao sauvage que l'on venait autrefois récolter depuis Cayenne ». Selon Patris la nation Roucouyenne est considérable, divisée en six peuplades, plus ou moins éloignées, mais il n'a pu en rencontrer que trois durant ce premier voyage. Il y dénombre 70 à 80 guerriers armés d'arc et de flèches (Patris 1766-67 : 38).

1769 : Tony, lors de son deuxième voyage, séjourne chez les Roucouyennes durant trois semaines : Il décrit un grand village entouré de palissades avec une organisation militaire situé vers les sources du Marouini (Tony 1769).

1789 : Leblond voyage sur la haute Camopi et le haut Marouini. Il cite parmi les 33 villages visités, le village de Touarinké (Twenké) (Pouliquen 2001). Il s'agit très certainement du village situé à Mope sula qu'Haïwé nous a décrit comme étant l'ancien village de Twenké. C'est là que les Wayana ont recueilli les Aluku en 1793 (Haïwé comm. pers.) Ce village est effectivement situé non loin du village Akuba booko goo où s'étaient installés les Boni, alors sous la direction du chef Boni, et où ils se sont fait massacrer par les Ndjuka. 

siècle. Ils y restèrent au moins 200 ans jusqu'à leurs migrations le long des deux grands affluents du Maroni : le Litani et le Marouini. Le clan Upului et ses alliés descendent le Litani et arrivent dans la région des grands sauts du Litani (secteur de l'actuel village d'Antécume pata) vers 1900. Tandis que le clan Kukujana (Kukuyana) (celui de l'actuel Gaan Man) descend le Marouini. C'est seulement au milieu du $\mathrm{xx}^{\mathrm{e}}$ siècle qu'ils sédentarisent leurs villages à leurs emplacements actuels (Fleury et al. 2016).

\section{Les villages Boni/Aluku du Marouini}

Juini. Mais l'histoire des Boni telle qu'il la raconte dans son ouvrage (p.64-72) est totalement romancée et ne correspond pas à la tradition orale.

D'après le récit du Capitaine Cazal, cette attaque a eu lieu au village de Akuba booko goo près du saut du même nom. Marouini, devait abriter un autre groupe de Boni (peut-être sous la direction d'un autre 
chef Koomanti Kodjo ou Puja van Lapaix, tous deux également tués dans cette bataille contre les Ndjuka (Moomou 2013 : 236).

Lors de notre mission en 1999, nous avons relevé les emplacements de : (Figures 6 et 7)

- Boon konde

- Mope sula konde

- Akuba booko go sula konde

- Crique Koutou : lieu du traité de paix entre Wayana et Aluku

- Crique SInale (Chinalé)

Coudreau, quant à lui, signale la présence de huit villages dans le Marouini :

- un à Soualaoni (petite crique affluent du haut Marouini)

- un à l'embouchure du Marouini, rive gauche (donc non loin de l'actuel emplacement d'Antécume pata)

- deux sur la crique Koutou (Kutu)

- deux à Ehnanticaye (près du saut Wajo, sur la carte de Coudreau, non loin de Mope sula : village d'Akuba booko go?)

- un en amont de la crique Ikoutou ${ }^{14}$

- un en aval de Baca Campou (baka Kampu) à deux heures en bas du Ouanapi.

Les différents villages ont pu être situés sur la figure 6 en confrontant la carte établie par Coudreau ${ }^{15}$ à nos propres relevés. Mais certains villages cités par Coudreau ont été oubliés par la mémoire orale.

En 1815 les Aluku quittent le Marouini, certainement las de l'isolement. Le Gaan Man Agosu (fils de Boni) vient de mourir. Il a été remplacé par Gongo, qui prend la décision de quitter la rivière, certainement pour se rapprocher des lieux de trocs commerciaux. Il vivra jusqu'en 1841 , date à laquelle il se fait tuer en tentant un rapprochement de l'Oyapock (Price \& Price 2003).

D’après le capitaine Cazal (cf. encadré)

« Les premiers villages furent fondés près d'Awala Soula (Santi goo ${ }^{16}$ ) près du vieux village Tiliwé. Il y eut un village à l'emplacement actuel de Maripasoula (derrière la mission catholique) marqué par un faaga tiki ${ }^{17}$. Puis ils fondèrent les villages de Kormontibo et de Cottica. Kormontibo servait de poste de garde contre une incursion éventuelle des Indiens. Le gros de l'effectif était à Cottica. On croit savoir que c'est Aluku qui aurait fondé ce village ».

Cependant en 1815, Aluku aurait eu plus de cent ans, car il est né dans les années 1710. Restent donc deux hypothèses :

- Soit le village de Cottica a réellement été fondé par Aluku, mais avant l'installation sur le Marouini, c'est-à-dire en 1791, pendant que Boni occupait le village de Pampu goõ ( pampu/giraumon; goõ ou goon/abattis) à Gaan day d'où il a attaqué les Ndjuka à Animbaw en 1792. En effet il est précisé dans les textes que les effectifs des Boni n'étaient jamais rassemblés dans le même village, pour plus de sécurité. Boni et Aluku devaient donc avoir chacun leur village. (Période « Lawa 1 » sur la figure 5)

- Soit on sous-entend que ce sont les descendants d'Aluku qui créent le village en 1815 ?

Nous penchons pour la première hypothèse, car Cottica, est réellement considéré comme le plus ancien village. Et les Boni/Aluku, pratiquant le culte des Ancêtres, sont très attachés à leurs cimetières et leurs lieux de prière. De plus, actuellement n'y résident plus que les membres du lo jakobi (c'est-à-dire le lignage d'Asikan-Sylvester et d'Aluku qui étaient donc parents). D'ailleurs cette hypothèse est confirmée par Delafosse (Gouverneur 
honoraire des colonies), qui nous dit que les Boni lors de leur première remontée du Maroni, fondent deux villages fortifiés : Bonidoro et Cottica (Delafosse 1925).

Essayons à présent de dater la réinstallation des Boni sur leurs lieux de vie actuels en croisant sources orales et écrites.

\section{Hypothèse sur l'histoire du peuplement du Lawa (Figure 5)}

D'après les sources orales, notamment chez les Wayana, des villages auraient été fondés sur le Litani, sans qu'ils ne soient précisément datés. Ainsi un village Boni/Aluku était installé à l'actuel emplacement du village de Taluwen (Opoya). Ce village avait été créé par Solati, qui avait aussi un autre petit village en face (Haïwé comm. pers.). Ces villages datent certainement du début $d u \mathrm{xx}^{\mathrm{e}}$ siècle, car Solati aurait vécu dans les années 1900-1950.

Ceci expliquerait pourquoi Twenké lors de son arrivée sur le Litani, dans les années 50, s'est installé sur une île inondable et non pas sur l'emplacement plus propice car en hauteur, de l'actuel village de Taluwen : les Boni y avaient établi des habitations. Or tous les riverains respectent la loi du primo-arrivant. Celui qui défriche possède une autorité sur le lieu, et nul autre ne peut s'y installer sans son autorisation.

De même les Boni ont longtemps eu une habitation à l'emplacement de l'actuel village d'Antécume pata (sur l'îlet Tolinga), où le grand man Tolinga a continué à résider régulièrement jusqu'à sa mort, en 1990. Cognat a dû demander l'autorisation au grand man Tolinga pour installer son village à Antécume pata en 1967 (Cognat comm. pers.). Ces faits soulignent une occupation de cette région par les Boni avant l'installation des Wayana.

Quels villages ont été créés en 1815, quand les Aluku quittent le haut Marouini, pour se rapprocher des centres d'échange et de commerce? Toujours sous la tutelle de leurs ennemis Ndjuka, ils s'installent dans la région du Lawa comme écrit dans les textes, mais certainement sur le haut Lawa c'est-à-dire du côté de Lawa mofu (embouchure du Lawa), emplacement actuel du village d'Anapaïké. Remarquons que ce village est toujours appelé Lawa par les Wayana. Selon le capitaine Haïwé, qui l'a lui-même appris du Gaan Man Tolinga, les Boni l'avaient baptisé « Cottica », d'où une certaine confusion avec le village situé sur le bas Lawa. Il existait aussi un autre village, rive française cette fois, qui s'appelait Agoodé (nom de l'actuel village de Boniville), non loin du lieu-dit santi goon ( santi/sable; goon/abattis), près de l'ancien village de Tiliwe (Haïwé comm. pers.). Ce témoignage rejoint celui du capitaine Cazal recueilli par Jean Hurault. 
Figure 10 : Village Boni le long du Maroni dans les années 1960

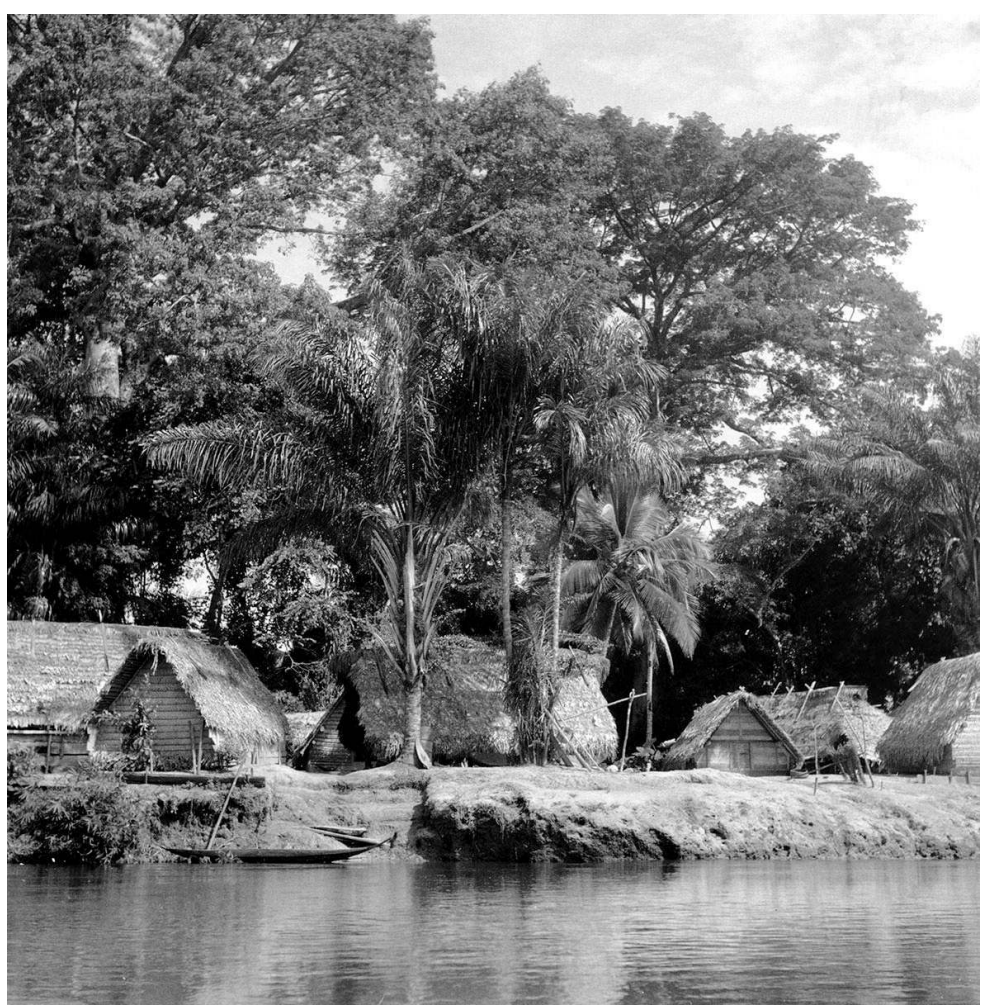

(c) J Hurault (Hurault 1961) l'embouchure de l'Inini où ils ont toujours des terres lignagères (Hurault 1961 : 109). Mais ils ne souhaitaient peut-être pas se rapprocher davantage de leurs ennemis Ndjuka installés sur le Tapanahoni. Cette hypothèse expliquerait aussi leurs rapports avec les Oyaricoulets, alors installés sur l'Ulemali (affluent du Litani, $c f$. figure 6). Après des débuts cordiaux, ces rapports se terminèrent par la guerre vers 1845 (Price \& Price $2003: 42$ ). installation sur le bas Lawa où ils résident encore (entre Cottica et Maripasoula) un peu plus tard, peut-être vers 1850-860, comme le confirme l'historien Jean Moomou (Moomou 2004, 2013 : 187). Le lieu de vie des Aluku entre 1815 et 1850-60 serait donc celui des actuels Wayana (sur le Litani), de l'embouchure du Lawa (voire du Marouini) jusqu'à l'embouchure de l'Inini (vers l'actuel bourg de Maripasoula). Cette version est confirmée par un des rares témoignages de l'époque. En 1832 quand le pharmacien Leprieur cherche à joindre les sources du Maroni, il sait par une précédente expédition que «les Boni occupent le confluent de l'Inini et du Lawa ». Lui-même les rencontre entre le confluent du Tampok et Maripasoula (Hurault $1960: 110)$. Coudreau (1893: 84) nous signale aussi que les Boni ont descendu du Marouini dans la haute Aoua (Lawa) puis dans la moyenne Aoua (Lawa). Les Boni descendent donc progressivement le fleuve Maroni.

Quelques villages et campements (kampu) existaient encore dans cette région du haut Maroni, lors de mon arrivée sur le fleuve en 1986, notamment à Kumakapan (en face de l'actuel village wayana de Kumakapan ${ }^{18}$ ), et à Awala sula dans le saut du même nom. Le campement de Kumakapan a totalement disparu ${ }^{19}$, et le village d'Awala sula est actuellement plus ou moins abandonné. 
Quand le capitaine Cazal mentionne l'installation des Aluku dans la région d'Awala sula, il est probable que cette installation ait duré quelques dizaines d'années sur cette portion du haut Lawa (où les Wayana ne sont arrivés qu'au milieu du $\mathrm{xx}^{\mathrm{e}}$ siècle).

\section{La réinstallation sur le bas Lawa}

3 Entre 1790 et 1860 les Ndjuka ont installé un barrage sur le fleuve, empêchant tout voyageur d'entrer en contact avec les Boni ${ }^{20}$ et tout Boni de descendre l'aval du fleuve Maroni. Vers 1830 les Boni, las de la tutelle des Ndjuka, tentent de s'installer sur l'Oyapock, mais cette tentative se solde par un échec et la tuerie de Cafesoca en 1837 puis la mort du Gaan Man Gongo en 1841 (Price \& Price 2003).

La tutelle des Ndjuka est levée en 1860 (Ronmy 1861), et les Aluku sont alors libres de voyager comme bon leur semble. C'est en 1890 que le Lawa est reconnu comme frontière officielle entre les deux Guyanes et les Boni choisissent de vivre sous l'autorité française dès 1891.

En 1887, Coudreau relève la présence de quelques villages Boni sur le bas Lawa :

Cottica, où réside le Gaan Man Anato

Ouecondo (weeko undo/à l'ombre du Pois sucré), appelé aussi PoMofou, situé sur un plateau élevé, avec vue sur le Lawa et la montagne Cottica, et où vit Aponchi (oncle d'Apatou).

Foowata (foo/quatre; wata/eaux) : ancien village du grand man Gongo, et de suite en amont :

Assissi et La Paix tous deux sur des îlots, sous la direction du capitaine Adam. Assissi est à l'époque le plus beau village Boni (Coudreau 1893). Notons qu'il est actuellement abandonné en raison des inondations fréquentes, les habitants sont allés rejoindre les habitants de Loka.

66 Le village de Loka a probablement dû être créé par les habitants de lî̀lot La Paix, pour les mêmes raisons. C'est en effet le lo Lapé qui réside à Loka, et anciennement à Assissi ede Kondé (« la tête du village d'Assissi »).

Les villages de Cottica, Lapé (Lapaix), Assissi et Kormontibo existaient déjà en 1861, puisque leurs noms figurent dans le rapport de la mission de délimitation francohollandaise (Hurault 1961). Selon J. Moomou (2013: 187) les villages de Cottica, Assissi, Puumofu et Kormontibo ont été créés entre 1840 et 1850.

68 C'est donc un peu avant la tentative de migration sur l'Oyapock (Gongo étant encore vivant) que les premiers campements se sont installés sur le bas Lawa. L'échec de cette tentative au début des années 1840 a dû les décider à se réinstaller sur leurs lieux de vie actuels, d'abord à Cottica, Assissi, Puumofu et Kormontibo, quelques années avant la levée du barrage des Ndjuka sur le Maroni en 1860.

69 Puis, en 1895, le Gaan Man Ochi fonde les villages de Agoode (Boniville), Loka, Tabiki (L'enfant perdu), et Papaïchton (Othily $1988: 14$ ).

70 Ces villages, connus encore aujourd'hui, regroupent chacun un ou deux matrilignages (lo ):

Boniville/Agoode : lo Dikan (le lo de Boni, actuellement éteint)

Cottica : lo Jakobi (le lo de Asikan-Sylvester et Aluku)

Papaïchton : los Dipelu et Kawina (lo de Koomanti-Kodjo, et Kwadjani) 
Kormontibo : lo Dipelu

Apatou : lo Dipelu

Tabiki (en face de Kotica) : lo Dipelu

Assissi bilo kondé : lo Dipelu

Assissi ede konde : lo Lapé (lo de Puja van Lapaix)

Assissi midi konde : lo Dju (cœur de l'île/île qui appartient à ce clan. Les autres sont venus après).

Loka : lo Lapé. (Hurault 1961, Moomou 2013)

\section{Vers l'autochtonie}

71 D'esclaves échappés à hommes libres, longtemps considérés comme des réfugiés (Hurault 1960, 1961), les Boni/Aluku ont vu leur statut évoluer en même temps que leur rapport au territoire.

La découverte de l'or, par le Gaan Man Anato en 1885 dans la région du Lawa a eu un rôle important. Faute de pouvoir faire l'exploitation lui-même, il en tire des bénéfices non négligeables grâce au commerce et aux taxes qu'il ponctionne sur l'or extrait dans la région. L'administration française décide ensuite de créer le territoire de l'Inini en 1930, puis le centre administratif de Maripasoula en 1950, afin de mieux réglementer cette activité. En 1946, avec la départementalisation, le territoire de l'Inini est transformé en arrondissement (Hurault 1972). À la création des communes en 1969, les Boni/Aluku sont devenus des citoyens français à part entière et le Gaan Man Tolinga est alors le premier maire de celle qui regroupe Apatou, Grand-Santi et Papaichton. Papaichton, décrétée commune en 1992, est considérée aujourd'hui comme la « capitale du pays Boni » comme indiqué sur le panneau au dégrad d'arrivée en pirogue sur le bourg.

Habitants du Parc Amazonien de Guyane au même titre que les Amérindiens, les Aluku partagent aussi avec les Wayana, une Zone de droit d'usage collectif sur le haut Maroni (Commune de Maripasoula). Par décret ministériel en 1987, les ZDUC sont réservées aux « communautés d'habitants qui tirent traditionnellement leurs moyens de subsistance de la forêt » c'est dire qu'on leur reconnaît une amorce à des droits territoriaux (Davy et al. 2012 : 8), le partage d'un même territoire avec les Amérindiens et par là-même un statut assimilé à celui d'Autochtones. Les rapprochements politiques entre Amérindiens et Noirs marrons se sont encore manifestés très récemment pendant le mouvement social historique qui a secoué la Guyane en mars et avril 2017 puis dans la mise en place du grand Conseil Coutumier en février 2018. Ainsi, depuis leurs premiers combats pour la liberté jusqu'à l'époque contemporaine, l'histoire des Aluku est intimement liée au territoire, et à leur histoire croisée avec celle des Wayana.

\section{Conclusion}

Cette étude, menée principalement en discussions animées autour de la carte et sur les lieux des événements du passé, nous a permis de mieux localiser les anciens lieux de vie des Wayana et des Boni sur le Marouini, même si certaines données restent à préciser. Soulignons l'importance des sauts dans la conquête du Maroni pour les Boni et du Litani pour les Wayana. Dans l'histoire des Boni, le passage de grands sauts après des évènements guerriers était une protection contre les forces militaires hollandaises. Ainsi 
les différentes étapes de la conquête du Maroni par les Boni se déroulent toujours par le franchissement des grands sauts (Figure 9):

- le saut Hermina après la première période (1776-1783) sur le bas Maroni (Sparouine) ;

- le saut Abounasunga après la deuxième période (1784-1790) de Bonidoro jusqu'à

l'embouchure du Tapanahoni ;

- les grands sauts du Marouini après la période de Pampu goon (1790-1792).

Ces grands sauts du Marouini (kassaba sula, Nassau dede sula, gaan kubi sula, et puu sani sula) sont toujours de véritables barrières à passer. Ils constituaient des obstacles non négligeables à franchir pour les ennemis. Les Aluku y font toujours une libation et une prière pour protéger leur passage, et invoquer leurs ancêtres.

Remarquons également que l'établissement près des sauts permet de garantir une bonne pêche, ces zones étant très poissonneuses.

77 La rencontre des Wayana et des Boni a été importante pour les deux peuples : Elle a été décisive dans l'histoire des Wayana, car elle leur a permis d'acquérir la technique de fabrication des canots, leur donnant les moyens de franchir les grands sauts, et ainsi descendre les rivières jusqu'à leur lieu de vie actuel (Fleury et al. 2016). Elle est extrêmement importante aussi pour les Boni, car c'est grâce aux Wayana qu'ils ont pu survivre dans l'extrême sud de la Guyane (à Mope sula et sur la crique Sinalé), où ils ont été recueillis par les Wayana avant de reconstruire de nouveaux villages.

Cet échange est souligné par les deux groupes par le terme alukujana, avec lequel ils s'auto-dénomment parfois, et qui symbolise la rencontre des deux peuples. Il a été scellé par un pacte de paix noué sur la crique Koutou en 1815, avant le départ des Aluku de cette partie de la rivière. Il est important de constater que malgré leurs divergences sur l'histoire, et leurs formes d'organisations sociales particulièrement contrastées, un respect mutuel entre Wayana et Aluku a toujours été de mise.

79 Une carte historique du Marouini et du haut Maroni, donne des éléments pour mieux contextualiser des épisodes importants de l'histoire de ces deux peuples, mais aussi les usages contemporains de l'espace. Les enjeux territoriaux sont toujours d'une extrême importance dans un contexte de conflits d'usages, comme on peut le constater actuellement notamment avec l'importance de l'orpaillage illégal qui affecte à nouveau cette région. La création du Parc national (Parc Amazonien de Guyane créé en 2007) a été également l'occasion de nombreuses réunions entre Boni/Aluku, Wayana et Administrateurs de l'État français. Les échanges pouvaient être particulièrement vifs, comme lors d'un comité de pilotage, où les Boni/Aluku voulaient exiger du parc l'interdiction pure et simple aux Ndjuka de venir sur leurs territoires ancestraux. La mise en place d'un parc avec un cahier des charges spécifique, faisait craindre la remise en cause des accords historiques traditionnels, au point même d'envisager de retirer cette région du haut Marouini du territoire du Parc. Les Aluku/Boni fermement opposés au Parc dans ses débuts, avaient par ailleurs mobilisé la figure de la carte tout en revendiquant, comme on l'a déjà vu, un droit sur le sol de primo-occupant «nous étions déjà sur le Haut Maroni quand vous nous y avez trouvé grâce aux cartes ». (Fleury 1998).

Cette histoire du Marouini est donc extrêmement importante dans la culture aluku et cet affluent du haut Maroni, qu'ils appellent Gaan Mawina, est un lieu de sanctuaires ou les Gaan Man aluku successifs viennent régulièrement faire des pèlerinages et des libations.

81 Soulignons l'importance de la carte comme objet politique, quand elle intervient comme médiateur lors de situations conflictuelles dans les décisions d'aménagement (Robert \& 
Duvail 2016). Les cartes, plus encore peut-être les cartes historiques, qu'elles soient ou non établies avec une méthode participative, sont susceptibles de ranimer des conflits territoriaux et doivent donc être soigneusement contextualisées. Mais, en permettant aussi de replacer l'occupation des terres dans le temps et dans l'espace, elles offrent aux jeunes générations, la possibilité de se replonger dans leur histoire, et dans le fondement même de leurs relations au territoire guyanais.

Enfin, il faut dire que les enjeux et les conflits susceptibles d'être mobilisés autour des cartes ne sont pas seulement manifestes à l'échelle locale. N'oublions pas qu'à cette époque du marronnage, les frontières entre les États coloniaux n'étaient pas encore tracées, et qu'elles étaient même l'objet de conflits entre la Hollande et la France (territoire contesté entre le Lawa et le Tapanahoni). C'est seulement en 1861 que le Lawa fut considéré comme la frontière officielle. Les Boni choisissant l'État français, quittèrent le «bord» hollandais du fleuve (sauf à Cottica ${ }^{21}$ ). Les populations riveraines ont joué un rôle important dans ces rapports entre Français et Hollandais de l'époque qui cherchaient à peupler leurs colonies, et les Aluku/Boni n'en sont pas dupes. Ils ont donc joué un rôle essentiel dans l'établissement des frontières entre les deux Guyanes.

Tradition historique des Boni par Jean Hurault

Notes prises en 1962 d'après un récit du Capitaine Cazal, qui a recueilli ces traditions de son père.

Boni aurait eu environ 50 ans au moment de la révolte. Il aurait eu un obia très fort qu'il avait obtenu au prix du meurtre d'un jeune homme.

On ne sait plus rien des combats sur la rivière Cottica. On sait seulement que dans la phase finale, en rejoignant le Maroni, les Boni se déplaçaient en brousse, faute de canots. En arrivant au fleuve près de la crique appelée Boni kiiki près d'Albina, ils tuèrent un indien Galibi, pour prendre son canot.

Les Boni s'établirent (cf. figure 5)

- d'abord près du lieu-dit St Maurice, puis remontèrent progressivement :

- sur la crique Serpent

- sur la Sparouine

- à Bonidoro (combat à Ablafo)

- à Yaloua (crique Salvat actuelle. Ce village est encore marqué par des poteries abandonnées. - à Ampuma (Poku tabiki). C'est là que les Boni auraient eu contact avec les Djuka. Boni leur aurait vendu l'Obia Kumenti, en échange d'une femme, Pikinbosi ${ }^{22}$ (car en ce temps-là on échangeait les femmes selon la coutume africaine).

- à l'embouchure du Tapanahoni

- à Gaã Day près d'Abunasunga. Ce village est encore marqué par les poteries abandonnées et la base d'un fraga tiki. On en a érigé un neuf et le grand-man y va prier de temps à autre. Ce village aurait été appelé Pampu goõ ${ }^{23}$ parce que les Boni affamés, n'ont pu subsister que par les giraumons, premier légume récolté dans les abattis.

On se souvient que dans ces déplacements successifs, beaucoup de gens périrent de faim et de misère, ne pouvant récolter leurs abattis abandonnés. Boni décidait de ces déplacements soudainement, et il fallait s'exécuter séance tenante. Il tuait de sa main ceux qui s'opposaient à ses ordres. Il était devenu d'un despotisme extrême. On dit même qu'il faisait tuer les enfants en bas âge qu'il jugeait incapables de suivre.

Les Boni n'avaient alors que de petits et mauvais canots, et pour transporter tout le monde il fallait de nombreux va-et-vient. 
À Gaã Day, ils auraient été l'objet d'une députation des Hollandais par l'intermédiaire de l'Indien Meku. On leur aurait proposé la paix en échange de leur soumission et de la présence parmi eux d'un administrateur blanc.

Boni aurait refusé.

Il décida ensuite, pour des raisons demeurées obscures, d'attaquer les Djuka et d'enlever l'administrateur hollandais établi parmi eux à Pukéti. Partant de l'emplacement actuel du village Alassa, ils passèrent par la brousse et attaquèrent le village du grand man Djuka (Pukéti) massacrant toute la population. Mais le grand man et l'administrateur blanc échappèrent et ils durent se retirer. On se souvient de quelques anecdotes de ce combat : un vieillard lépreux qui s'était caché dans une case, abattit d'un coup de fusil le porte-clairon des Boni.

Une femme enceinte avait demandé qu'on l'épargnat, mais Boni la fit tuer à son tour. Menacées désormais par les Djuka, les Boni s'établirent sur la rivière Marouini, près du saut Akuba booko goo. Ils y eurent la révélation du culte Tata Udu, et qu'ils reçurent ses lois interdisant la violence et la sorcellerie.

C'est là qu'ils furent attaqués par les Djuka. Ceux-ci surprirent les deux guetteurs placés en aval, nommés Pinki et Yanu. Le premier refusa de se rendre et fut tué. Le second accepta moyennant la vie sauve, de les conduire jusqu'à Boni. Ils passèrent un second poste de garde commandé par Atopa ${ }^{24}$, fils de Boni, qui dormait et ne les vit pas, et arrivèrent par surprise au village de Boni. Un combat s'engagea. Un second poste de guet commandé par Akese, qui veillait en amont contre les Indiens, intervint, mais les Boni qui n'avaient plus de munitions furent presque tous tués ou capturés. Beaucoup de Djuka perdirent la vie également. On raconte que Boni avait été trahi par sa femme Pinkinbosi à qui il avait révélé qu'on ne pouvait le tuer qu'à coups de marteaux sur la tête. Les Djuka coupèrent la tête de Boni et redescendirent la rivière dans deux canots.

Mais ils coulèrent à Dagu édé et perdirent la tête. Ils la remplacèrent par celle d'un des leurs tué au combat pour toucher la prime versée par les Hollandais.

Une partie des Boni dirigée par Aluku, beau-frère de Boni avait refusé le combat et s'était enfui en amont. C'est lui qui aurait sauvé les Boni d'une extermination complète, c'est pourquoi les Boni se disent Aluku pikin, les enfants d'Aluku.

Sous le commandement d'Atopa (Agosu) fils de Boni, les survivants s'étaient retirés dans l'extrême Sud à proximité de la crique Sinalé. Personne ne les y poursuivit, et une quinzaine d'années plus tard, ils revinrent s'établir en aval. Ils étaient passés sous la tutelle des Djuka qui ne leur livraient qu'en petite quantité les produits dont ils avaient besoin, contre d'importantes livraisons, de canots notamment.

Les premiers villages furent fondés près d'Awala Soula (Santi goõ, près du vieux village Tiliwé).

Il y eut un village à l'emplacement actuel de Maripasoula (derrière la mission catholique) marqué par un faaga tiki.

Puis ils fondèrent les villages de Kormontibo et de Cottica. Kormontibo servait de poste de garde contre une incursion éventuelle des Indiens.

Le gros de l'effectif était à Cottica. On croit savoir que c'est Aluku qui aurait fondéce village.

Le récit du capitaine Cazal nous a apporté des éléments importants de l'histoire des Boni, qu'on croyait effacés de la mémoire des Anciens. Soulignons aussi le rôle du travail de Jean Hurault dans le recueil de la mémoire orale des Aluku (Boni) et l'importance des sources orales aluku et wayana dans la reconstitution de leur propre histoire. Qu'ils en soient ici chaleureusement remerciés. Je remercie également sincèrement Pascale de Robert, Jean Moomou, Pierre Grenand et les 
relecteurs anonymes de la Revue Ethnoécologie pour la relecture critique de cet article, et Pierre Joubert, cartographe au Parc Amazonien de Guyane, pour la réalisation de la figure 2.

\section{BIBLIOGRAPHIE}

Bastide R. 1967 - Les Amériques noires. Paris, Payot 236 p. (Petite Bibliothèque Payot).

Chapuis J. \& Rivière H. 2003 - Wayana eitoponpë. (Une) histoire (orale) des Indiens Wayana. Matoury, Éditions Ibis rouge, 1065 p. [En ligne] : http://classiques.uqac.ca/contemporains/chapuis_jean/ Wayana_eitoponpe/Wayana_eitoponpe.pdf

Chapuis J. 2007 - L'ultime fleur. Ekulunpï tihmelë. Essai d'ethnosociogénèse wayana. Encyclopédie wayana. Orléans, Presses Universitaires Orléans, 164 p.

Coudreau H. 1893 - Chez nos Indiens. Quatre années dans la Guyane française (1887-1891). Paris, Hachette, 609 p.

Crevaux J. 1876-1879 (Rééd.1987) - Le mendiant de l'Eldorado. Paris, Phebus, 413 p. (Collection d'ailleurs).

Davy D., Tritsch I. \& Grenand P. 2012 - Construction et restructuration territoriale chez les Wayãpi et Teko de la commune de Camopi, Guyane française, Confins [En ligne], 16 | 2012, mis en ligne le 16 janvier 2013, consulté le 20 juin 2017. URL : http://confins.revues.org/7964 ; DOI : 10.4000/confins.7964

Delafosse M. 1925 - Survivances africaines chez les Nègres « Bosch » de la Guyane. L'Anthropologie (35) : 475-494.

De Groot S. 1984 - La guerre des Marrons Boni (1765-1793). Équinoxe 19 : 1-29.

Dupuy F. (Ed.) 2012 - Les arpenteurs des confins. Explorateurs de l'intérieur de la Guyane (1720-1860). Paris, CTHS, 290 p. (La librairie des cultures ; 4).

Dupuy F. 2015 - Noirs marrons et Amérindiens des Guyanes : approche comparée des relations interethniques. In : Moomou J., APFOM (Ed.) Sociétés marronnes des Amériques. Mémoires, patrimoines, identités et histoire $\mathrm{du} \mathrm{XVII}^{\mathrm{e}}$ au XX $\mathrm{X}^{\mathrm{e}}$ siècle. Matoury, Ibis rouge Éditions : 269-279.

Fleury M. 1991 - "Busi nenge", les Hommes-Forêt. Essai d'ethnobotanique chez les Aluku (Boni) en Guyane française. Thèse présentée pour l'obtention du titre de docteur de l'université Paris 6 , spécialité : Biologie Végétale Tropicale, $357 \mathrm{p}$.

Fleury M. 1996 - Plantes alimentaires et identité culturelle chez les Marrons Boni (Aluku) de Guyane française. In : Hladik C.M., Hladik A., Pagezy H., Linares O., Koppert G.J.A. \& Froment A. (Ed.) L'Alimentation en forêt tropicale. Interactions bioculturelles et perspectives de développement, 2 vol., Paris, Unesco MAB, L'homme et la biosphère : 973-984.

Fleury M. 1998 - Les populations du haut-Maroni et le projet de parc national de la Guyane. JATBA, Revue d'Ethnobiologie 40 (1-2) : 577-610.

Fleury M. 1999a - Maripasoula. Premiers villages aluku du Maroni. In : DRAC Guyane. Service archéologique Bilan scientifique (année 1999). Recherches ethno-archéologiques. 
Fleury M. 1999b - Dénomination et représentation des végétaux en forêt tropicale : étude comparative chez les Amérindiens wayana et les Noirs marrons aluku de la Guyane française. In : Bahuchet S, Bley D., Pagezy H. \& Vernazza-Licht N. (Ed.) L'Homme et la forêt tropicale. Ed de Bergier, SEH : 31-44.

Fleury M. 2010 - Du mythe de la nature au mythe du monde moderne : représentation du monde et modernité chez les Amérindiens wayana. In : Amaz'hommes. Sciences de l'homme et sciences de la nature en Amazonie. Matoury, Ibis Rouge Éditions : 217-234.

Fleury M., Alupki T., Opoya A. \& Aloïké W. 2016 - Les Wayana de Guyane française sur les traces de leur histoire. Revue d'ethnoécologie [En ligne] 9, 2016, mis en ligne le 01 juillet 2016, consulté le 05 juillet 2016. URL : https://ethnoecologie.revues.org/2711

Hoogbergen W.S.M. 1985 - De Boni-Oorlogen, 1757-1860, Marronage en guerilla in Oost-Suriname. Utrescht, Centrum voor Caraïbische Studies, $523 \mathrm{p}$.

Hoogbergen W. 1990 - The Boni maroon wars in Surinam. Leiden, Brill, 254 p.

Hoogbergen W.S.M. 2008 - Frères et ennemis Aluku et Ndjuka de 1710 à 1860 In Collomb G. \& Jolivet M.-J. (Ed.) Histoires, identités et logiques ethniques : Amérindiens, Créoles et Noirs Marrons en Guyane. Paris, CTHS, $221 \mathrm{p}$.

Hurault J. 1960 - Histoire des Noirs réfugiés Boni de la Guyane française. Revue d'histoire d'OutreMer 47 (166) : 76-137.

Hurault J. 1961 - Les Noirs réfugiés Boni de la Guyane française. Dakar, 363 p. + pl. (Mémoire de l'Institut Français d'Afrique Noire (IFAN) ; 63).

Hurault 1972 (rééd. 1989) - Français et Indiens en Guyane. Cayenne, Guyane, Presse Diffusion, 223 p. Hurault J., Grenand F. \& Grenand P. 1998 - Wayana et Wayãpi de la forêt. Bondy, IRD, 198 p. (Autrement).

Moomou J. 2004 - Le monde des Marrons du Maroni (1772-1860). La naissance d'un peuple : les Boni. Matoury, Ibis rouge, $216 \mathrm{p}$.

Moomou J. 2013 - Les Marrons Boni de Guyane. Luttes et survie en logique coloniale (1712-1880). Matoury, Ibis rouge, $597 \mathrm{p}$.

Othily A. 1988 - Éléments de chronologies Aluku.. Cayenne, ORSTOM, 16 p. (Série Dynamismes sociaux et développement).

Patris J.-B. 1766-1767 - Relation abrégée d'un voyage fait dans l'intérieur de la Guyane française en remontant l'Oyapock, le Camoupy etc., et descendant le Marony, après avoir pénétré dans les terres Presque jusqu'aux sources de ce dernier fleuve [1766-1767]. In Dupuy F. (Ed.) 2012 Les arpenteurs des confins. Explorateurs de l'intérieur de la Guyane (1720-1860). Paris, CTHS, (La librairie des cultures ; 4).

Poulalion J.-L. 1986 - Histoire du Surinam des origines à l'indépendance. La Chapelle Montligeon, Imprimerie de Montligeon, $93 \mathrm{p}$.

Pouliquen M. 2001 - Les voyages de Jean-Baptiste Leblond, médecin naturaliste du roi. 1767-1802. Antilles, Amérique espagnole, Guyane. Paris, CTHS, 340 p. (Format ; 47).

Price R. \& Price S. 2003 - Les Marrons. Vents d'ailleurs, 127 p. (Cultures en Guyane).

Price R. 2013a. - Les Premiers temps. La conception de l'histoire des Marrons Saamaka. Vents d'ailleurs, $300 \mathrm{p}$. 
Price R. 2013b - The Maroon Population Explosion: Suriname and Guyane. Research note. New West Indian Guide 87 : 323-327.

Robert P. de \& Duvail S. 2016 - « Mettre en carte » le territoire. Revue d'ethnoécologie [En ligne] 9 | 2016, mis en ligne le 01 juillet 2016, consulté le 20 juin 2017. URL : http:// ethnoecologie.revues.org/2739

Ronmy 1861 - Excursion dans le haut Maroni (Guyane française). Revue maritime et coloniale 1 : 779-796.

Stedman J.G. 1799-1800 (rééd. 1989) - Capitaine au Surinan. Une campagne de cinq ans contre les esclaves révoltés. Paris, Sylvie Messinger, 343 p. (Les pas de Mercure).

Tony 1769 - Voyage dans l'intérieur du continent de la Guyane chez nos Indiens Roucoyens. In Dupuy F. (Ed.) 2012 Les arpenteurs des confins. Explorateurs de l'intérieur de la Guyane (1720-1860). Paris, CTHS (La librairie des cultures ; 4).

Vidal G. 1861 - Voyage d'exploration dans le haut Maroni (Guyanes française et hollandaise). In Dupuy F. (Ed.) 2012. Les arpenteurs des confins. Explorateurs de l'intérieur de la Guyane (1720-1860). Paris, CTHS (La librairie des cultures ; 4).

Van der Kuyp E. 1961 - Overview of the literature on the diet and dietary habits on the Bushnegros of Suriname. Translated by Khepri Das. Niewe West-Indische Gids : 205-271.

Van Velthem L.H. 1998 - A pele de Tuluperê: uma etnografia dos trançados dos Wayana. Belém, Museu Paraense Emílio Goeldi, 251 p.

\section{ANNEXES}

Annexe 1 : Principaux toponymes relevés sur le Marouini en juillet 1999 (Figure 2) Kupi sula (« le saut de Kupi » en Aluku) : saut cité par Coudreau (1893 : 255).

= Tulala pata («village de tulala » en Wayana; tulala/Maranta ruiziana Körn (Marantaceae), plante dont le rhizome (toloman) est utilisé pour faire une fécule réputée faire grossir). Akuli sula (en Aluku) = Uluhani (en Wayana ; ulu/manioc $)$

Akuli (ou Acouli) est cité par Coudreau comme le Chef indien, grand chamane et gendre de Touanké. Il est le fondateur du premier village sur la crique Chinalé (Sinale). D'après l'auteur, d'origine apalaï, il aurait été le chef le plus influent du pays. Coudreau l'a emmené à Cayenne pour se faire consacrer « capitaine en chef des Roucouyennes français du Marouini et du Litani ", par les autorités françaises qui lui ont remis une canne et un diplôme comme insignes officiels de son grade (Coudreau 1893 : 109).

Sipali watop : campement wayana (sipali/raie ; watop/danse)

Alu patatpë : ancien village wayana d'Alu (patatpë/ancien village)

Pilëu pan (« lieu-dit des roseaux à flèches » en Wayana, pïlëu/roseau à flèche, Gynerium sagittatum (Poaceae) ; pan/lieu, endroit) : village habité en 1999 par des Apalai, venus du Paru.

Jamo pata : village habité en 1999 par des Apalai : la famille de Jamo le fils de Kutaka (qui vivait à Antécume mais décédé en 2006).

Pita pata : ancien village wayana

Ateteipë : ancien village wayana

Awalipaimë (lieu-dit du poisson géant : awalipa/poisson Guianacara spp., Chiclidae ; imë/ géant, sauvage) : crique impraticable, rive droite. 
Tulupele imë enï (lieu-dit du trou du Tulupele ${ }^{25}$ géant, tulupele/monstre qui empêchait les gens de passer la rivière ; imë/géant ; enï/trou, contenant). Ce même toponyme existe sur le Litani (Fleury et al. 2016), et sur le Jari et le Parou (Van Velthem 1998).

Tilokolokem (lieu-dit « terre blanche » en Wayana) : ancien site d'orpaillage où les Indiens travaillaient avec les Blancs.

Matatop (« le lieu du pourrissement»; mata/pourrir, être pourri) : lieu maudit, car autrefois de nombreux Indiens sont morts là : un homme les avait invités à danser, mais il était couvert de blessures, et personne ne voulait danser avec lui. Pour se venger, il fit mourir tout le monde (c'était un chamane qui a envoyé des maladies sur tous les habitants). Il y avait tellement de morts, qu'on ne pouvait pas les enterrer. Le toponyme signifie « le lieu du pourrissement », car les corps ont pourri sur place. (Interprétation : c'est très certainement un village décimé par une épidémie, due au contact des étrangers).

Asikalu waipu (asikalu/ canne à sucre ; waipu/jambière, genouillère de perles) : campement provisoire toujours employé par les Wayana. Autrefois Il y avait un beau village sur un plateau le long de la crique.

Tilopilem (lieu-dit « terre rouge ») : ancien village wayana, dont on a oublié le nom. Il y a beaucoup de bancs de sable, ou les Wayana viennent chasser les Iguanes.

Amaï kampu : ancien campement de Amaïpoti Touenke (fils de Twenke et actuel Gaan Man).

Malapi imë enï (lieu-dit « le trou de l'hirondelle géante » : malapi/hirondelle à ailes blanches (Tachycineta albiventer) ; imë/géant ; inë/trou) : grand saut en saison sèche. Ndjuka jalan (« le boucan des Ndjuka » en Wayana) sur une île : selon les Wayana, c'est là que les Boni et les Ndjuka se sont battus autrefois. C'est là qu'aurait eu lieu le massacre des Boni (là où les Ndjuka ont boucané, torturé les Boni).

Mekulakëhpan : crique rive gauche (mekulak = arbre ripicole dont les fruits sont mangés par les poissons, Triplaris weigeltiana (Rchb.) Kuntze, (Polygonaceae) ; pan/lieu en Wayana). Boon konde kiiki (« la crique du village brûlé » en Aluku) : emplacement de l'ancien village de Boni, sur la rive droite. Le village était très grand, il s'étalait tout le long de la rivière jusqu'à la crique Sihnatepan. Le village serait tombé dans la crique (Topo comm. pers.). Il été brûlé par les Ndjuka, lors de leur attaque des Boni. C'est pour cela qu'on l'appelle le « village brûlé » (Haïwé 1999).

Watau enï (Wayana) (watau/poisson coumarou (Tometes lebaili) ; enï/trou, contenant en Wayana) ou kumalu olo (kumalu/coumarou; olo/trou, en Aluku) ou doti olo (« trou de la terre » en Aluku) : c'est un trou très profond, entouré de rochers.

Tasikale patatpë (ancien village wayana de Tasikale), près du saut uliulu matop (saut en Wayana). Il y a eu également un ancien village d'orpailleur créole (Dizel).

Onotapoto : roche où l'on préparait les boules de roucou (onot en Wayana).

Sihnatëhpan (crique) (« sihnat/liane; pan/lieu, endroit) : lieu-dit « emplacement des lianes » en Wayana. « Le BRGM a travaillé là autrefois ».

Tëpuenetop (Wayana) ou Païpisibali (paï/beau-père ou gendre ; pisi/pisser ; bali/cri ou crier en Aluku) : saut où l'on aperçoit l'inselberg.

Konopuamoï (Roche Koutou) (en Wayana : tëpu/roche, inselberg ; enetop/où l'on aperçoit ; en Aluku nenge : konopu/bouton ; a moï/est joli « le joli bouton »).

Malipahpan (malipa/palmier maripa : pan/lieu, endroit : « emplacement des palmiers maripa » en Wayana) : ancien village wayana, rive droite.

Dizel kondé : ancien village d'orpailleur créole.

Panakali : gros rocher au milieu de la rivière [panakali est le nom wayãpi pour la vannerie 
nommée pamkalit en Wayana (P. Grenand comm. pers.)].

Langa sula (Aluku) : saut avec une île au milieu : langa sula tabiki (A.)

Kawatop (Wayana) = gaan sula (Aluku).

Sur la rive gauche se trouvait l'ancien village wayana Kawatop (lieu-dit « où dansent les poissons ») (ka/poisson; watop/danse).

Les Aluku y ont établi des petits campements provisoires. Kawatop aurait été le dernier village de Twenké.

Weti wata ( (eau blanche » en Aluku) = Wanapi $($ Wayana $):$ crique

Moï ston ( jolis rochers » en Aluku) = kuliputpë akulikatop (Wayana) : rochers sur la gauche de la rivière : endroit où les Wayana s'arrêtaient pour enlever la carapace des tortues (kuliputpë/tortue ; akulika/casser, fendre ; top/suffixe de lieu).

Kutu (crique rive droite) : c'est là que les Boni et les Wayana ont fait un traité de paix (sweli).

Uman gaan sula (uman/femelle ; gaan/grand ; sula/saut en Aluku) = Alimi egletop alimi/ singe atèle ; egletop/lieu où ils traversent en Wayana) : saut où les singes traversent en saison sèche (donc lieu privilégié pour la chasse aux atèles dont les Wayana sont très friands)

Wajo : Ancien village de Twenké l'Ancien. Selon les Wayana, c'est là qu'ils recueillirent les rescapés aluku du massacre de Ndjuka Jalan. C'est dans ce village qu'ils auraient caché une femme enceinte, qui aurait ensuite donné naissance à la descendance aluku. Mope sula (" le saut des mombins »): Saut où se trouvait l'ancien village boni/aluku Mope sula konde tout près du précédent village wayana Wajo.

Akuba booko goo sula (« le saut de la gourde cassée d'Akuba » en Aluku nenge) : Wijapan ( wija/salade coumarou (Mourera fluviatilis) ; pan/lieu, endroit : lieu-dit des salades coumarou (Wayana) : il y avait un village aluku sur la rive droite. (Selon le récit de Cazal, c'est là que Boni vint s'installer après l'attaque des Ndjuka à Puketi. Ils y eurent au pied d'un arbre (indéterminé) les révélations de Tata Udu, et reçurent ses lois interdisant la violence et la sorcellerie).

Silipipi imë enï : (silipipi/oiseau tyran féroce (Myarchus ferox, Fluvicoliné ; imë/géant ; enï/ trou) : trou d'eau où il y a beaucoup de wataa bubu (« tigres d'eau » en Aluku », monstres aquatiques) : c'est un endroit craint par les Indiens.

Sinale: embouchure de la crique où ont vécu les Wayana et les Boni (où se seraient réfugiés les rescapés du massacre par les Ndjuka).

Annexe 2 : Liste des Gaan Man Boni/Aluku avec la date de leurs règnes

Sylvester : $1712-1765$

Boni $1765-1793$

Agosu (Atopa) : 1793-1815

Gongo : 1815-1841

Adam : $1841-1870$

Atyaba (lo Jakubi) : 1870-1876

Anato (lo Jakubi) : 1876-1890

Ochi (lo Dikan) : 1891-1915

Awensaï (lo Dikan) : 1915-1936

Difou (lo Dikan) : 1937-1965

Tolinga : 1965-1990

Doudou (1991-2014) et Joachim (1991-présent) 


\section{NOTES}

1. Cet article fait partie des dossiers "Cartographie participative" publiés dans la Revue Ethnoecologie en 2017 et 2016"

2. De l'espagnol cimarron, signifiant le retour à l'état sauvage

3. Évaluation approximative, car on n'a pas de relevé exact de tous les membres de la communauté, incluant une diaspora importante.

4. Très certainement originaire des Kromanti de la Côte d'Or en Afrique (actuel Ghana).

5. D’origine écossaise (Price 2013a : 26).

6. La plantation « Groot Marseille » sur la rivière Cottica (Othily 1988).

7. (A.C., D.F.- Guyane 206, 1776, cité par De Groot 1984)

8. La fille du Chef Kwamina Adjubi, du lo Dikan (Hoogbergen 2008).

9. D'après Coudreau, vers 1785, Boni attaque Poketi, la capitale des Ndjuka, afin d'y enlever le représentant du gouvernement hollandais qui y résidait, dans le but de négocier sa libération en échange d'un traité de paix avec les Hollandais. Mais il se fait rattraper par les Ndjuka, qui se rangent alors du côté des Hollandais, inquiets de perdre leur liberté (Coudreau 1893 : 41-42). Cette version rejoint la version de Cazal enregistrée par Hurault en 1962.

10. L'identité du leader du groupe durant l'épopée du Marouini est parfois mise en doute, s'agissait-il toujours de Boni I, le fondateur alors âgé d'une soixantaine d'années, ou de Boni II connu aussi comme Boni Okilifu? (Moomou 2013). Pour les membres de l'expédition de 1999, c'est bien pendant les évènements du Marouini que meurt Boni le fondateur et pas avant, c'est pourquoi nous avons retenu cette version dans nos cartes et récits.

11. Dagu/chien; ede/tête: exprimant le mépris des Ndjuka de l'époque vis-à-vis de leurs ennemis Boni.

12. Appelée Atopala d'après les Wayana (Chapuis 2007).

13. Piquiolo de Coudreau (1893).

14. Il y a une certaine confusion entre les deux criques Koutout et Ikoutou, actuellement toutes deux appelées cirque Koutou sur les cartes-guides du Parc Amazonien de Guyane.

15. Nous tenons ici à remercier Pierre Grenand, qui nous a permis d'accéder à ce document.

16. Santi/sable; goõ ou goon/abattis.

17. «Autel des ancêtres» présent dans tous les villages traditionnels Aluku (konde), contrairement aux campements provisoires (kampu) qui n'en possèdent pas.

18. Kumaka/fromager, Ceiba pentendra ; pan/emplacement, lieu.

19. Ce campement (Kampu) était occupé par des membres du lo lapé du village de Loka et a été déserté à la mort de M. Dada en 1986. L'ensemble des habitants sont rentrés au village traditionnel (konde) pour les funérailles à Loka, et ne sont plus jamais remontés vivre sur le Kampu. Selon les Wayana, le campement aurait été incendié par la suite.

20. Ce qui explique le peu de témoignages écrits qui nous restent de cette époque.

21. Car on ne pouvait complètement abandonner ces terres ancestrales et les cimetières des Ancêtres.

22. Selon l'historien Wim Hoogbergen (2008), Boni avait épousé la fille du chef Ndjuka Kwamina Adjubi, du lo dikan.

23. Signifiant « l'abattis des giraumons ».

24. Appelé aussi Agosu

25. Cf. Mythe de Tulupele dans Van Velthem (1998) et Fleury et al. (2016). 


\section{RÉSUMÉS}

Cet article retrace l'histoire des Noirs marrons Boni également appelés Aluku et leur remontée $\mathrm{du}$ Maroni (fleuve frontière entre la Guyane française et le Surinam) pour fuir les militaires hollandais envoyés à leurs trousses. Leur destin a croisé celui des Wayana en provenance du Brésil, qui fuyaient les chasseurs d'esclaves armés par les Portugais, et s'étaient réfugiés aux sources du Maroni depuis plus de cent ans. Leur histoire croisée sur le Marouini, un affluent du haut Maroni a eu une influence très importante sur l'avenir des deux peuples. Un travail de cartographie a été réalisé avec des Anciens des deux communautés, sur les lieux de cette histoire mouvementée. Les cartes historiques issues de ce travail permettent de retracer les déplacements successifs des Anciens villages, et mieux connaître cette partie peu documentée de leur histoire.

The purpose of this paper is to trace the history of the Marroons Boni also called Aluku and their journey across the Maroni (boundary river between French Guiana and Surinam) to escape Dutch soldiers sent on their trails. Their destiny crossed the Wayana's path, from Brazil, who were trying to escape slaves' hunters, armed by the Portuguese, and had taken refuge at the sources of Maroni for more than one hundred years. Their crossed history on Marouini, a tributary of the Upper Maroni river, had a very important influence on the future of both populations. A map was elaborated with elders from both communities on the scene of this animated story. The ancient maps stemming from this work allow us to describe the successive migrations of the former villages, and better know this poorly documented part of their history.

\section{INDEX}

Index géographique : Marouini, haut Maroni

Population Wayana, Boni, Aluku

Keywords : Marouini, Upper Maroni, mapping, history, Wayana, Boni, Aluku

Mots-clés : cartographie, histoire

\section{AUTEUR}

\section{MARIE FLEURY}

Ethnobotaniste, Muséum National d'Histoire Naturelle (UMR MNHN-IRD 208 PALOC) 